\title{
On the Available Work Limits at Constant Heat and Entropy Production
}

\author{
Saeed Shahsavari ${ }^{*}$, Mehran Moradi $^{1}$, Morteza Esmaeilpour ${ }^{2}$ \\ ${ }^{1}$ Department of Mechanical Engineering, Isfahan University of Technology \\ Isfahan, Iran \\ ${ }^{2}$ Department of Civil Engineering, Geo and Environmental Sciences, Karlsruhe Institute of Technology \\ Kralsruhe, Germany \\ *Corresponding author's email: s.shahsavari [AT] me.iut.ac.ir
}

\begin{abstract}
In this paper, using the combination of the first and second laws of thermodynamics, the work bounds in thermodynamic cycles are investigated generally and, to show the application, the results are extracted for some physical systems. Also, a new concept on the a vailable work limits is extracted. To provide information on the maximum or minimum amount of work to be done during a thermodynamic cycle, energybalance, as well as irreversibility, should be considered. Entropyproduction during a thermodynamic cycle as a limiting criterion forwork to be done is expressed as Clausius inequality. Therefore an inequality extracted from the first and second laws of thermodynamic to obtain lower and upper bounds of available work. The obtained upper bound of the work to be done is in agreement with Carnot's rule. The lower bound is obtained at the maximum possible irreversibility during the respective cycle.
\end{abstract}

Keywords--- Thermodynamic cycle; Maximum work; Minimum work; Production Entropy;Maximumirreversibility

$\delta Q:$ System heat variation

$\delta w:$ SystemWork variation

T: Temperature

$T_{0}$ : Ambient temperature
R: Global constant of gases
c: Specific Heat
$\gamma$ : Thermal expansion coefficient
$I_{1}:$ First invariant of the stress tensor

\section{INTRODUCTION}

The upper bound work in thermodynamic cycles has been introduced by Carnot [1]. He introduced the irreversible processes and determined the maximum work can be done between two cold and hot temperatures. Therefore Carnotcan be known as the founder of the second law of thermodynamics. Based on the second law, the entropy of an is olated system will be increased always [2].

Another question can be raised on the upper work bound in the other systems as well as the lower work bound. Some researchers have investigated the work bound in dynamically loaded bodies [3]. Also, upper work bound in systems for multichannelqueues has been investigated [4]. The upper and lower bonds for free energy change have been determined [5]. And also, work bounds cyclically loaded creeping structures have been studied [6]. And the same researches on the other physical systems [7-10]. Als o, the dis sipation energy bounds can be determined due to viscous dissipation [11]. And the same works on the some physical systems [12-15].

In this report, the work bounds are investigated generally. According to the Clausius inequality, the term of $\oint \frac{\delta Q}{T}$ in a thermodynamic cycle may take any value in the period $(-\infty, 0]$ without any lower bound. A question may arise that is there any lower bound on this term or in other words, how negative it could be in a thermodynamic cycle. The other question is about the bounded values of available work. The concept of maximum work that can be doneis more familiar, but the amount of minimum work could be another problem. This question is in line with that one asks how many will be the maximum irreversibility that can occur in a thermodynamic cycle.

To answer these questions, a combination of the first and second laws of thermodynamic will be used as a means for bounding purposes. 


\section{CONCEPTS AND RELATIONS}

In a thermodynamic cycle, the threeterms $\frac{1}{T_{0}} \oint \delta Q, \frac{1}{T_{0}} \oint \delta w$ and $\oint \frac{\delta Q}{T}$ are expressed and related to each other in the first and second laws of thermodynamics.

The first law of thermodynamics [16]:

$\frac{1}{T_{0}} \oint \delta w=\frac{1}{T_{0}} \oint \delta Q$

The second law of thermodynamics [16]:

$\oint \frac{\delta Q}{T} \leq 0$

Combining thesetwo laws together gives:

$\frac{1}{T_{0}} \oint \delta w \geq \frac{1}{T_{0}} \oint \delta Q+\oint \frac{\delta Q}{T}$

$\frac{1}{T_{0}} \oint \delta w \leq \frac{1}{T_{0}} \oint \delta Q-\oint \frac{\delta Q}{T}$

The resultant relations are equivalent to the equations (1) and (2) and vice versa. In addition to the concepts of the first and second laws, these relations give information about the work done in a thermodynamic cycle and its irreversibility as well as its lower and upperbounds.

The following relation will result from these two relations:

$W_{\text {min }}=T_{0} \oint \frac{\delta Q}{T}+\oint \delta Q \leq \oint \delta w \leq W_{\max }=\oint \delta Q-T_{0} \oint \frac{\delta Q}{T}$

This equation defines the minimum and maximum av ailable work and is equivalent to the first and second thermodynamic laws. From this equation, two results can be concluded: $W_{\max }=\oint\left(1-\frac{T_{0}}{T}\right) \delta Q$ and $W_{\min }=\oint\left(1+\frac{T_{0}}{T}\right) \delta Q$. The first result is in accordance with Carnot's rule and determines the maximum work could be done [17], but the second result leads to a new concept:

\section{Lower bound of available work is when the irreversibility is at its upper bound.}

The minimum work is done, alsocan be rewritten as follows:

$W_{\min }=2 \oint \delta Q-\oint \alpha_{\max } \delta Q \leq \oint \delta w$

Where:

$\delta w_{\max }=\alpha_{\max } \delta Q$

That $\delta w_{\max }$ is the most work that may be done in the current state of the systemunder the effect of applied heat $\delta Q$. By using relation (7), the following equation could be obtained:

$\oint \frac{\delta Q}{T}=\frac{1}{T_{0}} \oint \delta Q-\frac{1}{T_{0}} \oint \alpha_{\max } \delta Q$

Equation (8) is an alternative formfor Carnot's rule about the maximum available work.

\section{APPLICATION}

The application of obtained inequality is investig ated for some examples, such as the ideal gases and an elas tic element.

\subsection{Ideal gases}

For ideal gases, the coefficient $\alpha_{\max }$ is earned as follows:

$\alpha_{\max }=\frac{R}{c_{p}}$

Where $c_{p}$ is the specific heat at constant pressure. For any thermodynamic cycle: 
$\oint\left(2-\frac{R}{c_{p}}\right) \delta Q \leq \oint \delta w \leq \oint \frac{R}{c_{p}} \delta Q$

Relation (10) shows the lower and upper bound for available work during different thermodynamic cycles. Relation (10) satis fies the first and second laws of thermodynamics.

In a reversible process, the minimumand maximum available works are equal; therefore, the following equations could be established:

$\oint\left(2-\frac{R}{c_{p}}\right) \delta Q_{r e v}=\oint \frac{R}{c_{p}} \delta Q_{r e v}=\oint \delta w_{r e v}$

So:

$$
\oint\left(1-\frac{R}{c_{p}}\right) \delta Q_{\text {rev }}=0
$$

Relation (12) is equivalent to the first and second laws of thermodynamics for reversible cycles.

\subsection{Elasticity loaded bodies}

For an elastic element, the coefficient of $\alpha_{\max }$ is earned as follows:

$\alpha_{\max }=\frac{\gamma}{c} I_{1}$

Therefore, the lower and upper bounds for available work are as follows:

$\oint\left(2-\left(\frac{\gamma}{c} I_{1}\right)\right) \delta Q \leq \oint \delta w \leq \oint\left(\frac{\gamma}{c} I_{1}\right) \delta Q$

Relation (14) shows the work bounds in elas ticity loaded bodies as well as dynamically bodies generally. Some researchers inves tig ated the work bound in dynamically loaded bodies by using other methods [3].

\section{CONCLUSIONS}

As, based on Carnot's rule, the work done in thermodynamic cycles has the upper bound, from combination first and second laws of thermodynamics, also can beresulted that the lower bound will exist. To determine a lower bound or upper bound for work done in a thermodynamic cycle, two factors should be considered simultaneously; energy balance as well as irreversibility. Therefore an inequality extracted from the combination of the first and second laws of thermodynamic to investig ate bounds of available work. The upper bounds obtained from extracted inequality are in agreement with the Carno's rule. The exis tence of a lower bound for available work indicates the existence of the upper bound for irreversibility in a thermodynamic cycle.

\section{REFERENCES}

[1] Erlichson, Herman. "Sadi Carnot,Founder of the Second Law of Thermodynamics'." European journal of physics 20.3 (1999): 183.

[2] Sheehan, D. P. "The second law of thermodynamics: Foundations and status." Foundations of Physics 37.12 (2007): $1653-1658$

[3] Ponter, A. R. S. "General dis placement and work bounds for dynamically loaded bodies." Journal of the Mechanics and Physics of Solids 23.2(1975): 151-163.

[4] Wolff, Ronald W. "Upper bounds on work in system for multichannel queues." Journal of applied probability 24.2 (1987): 547-551.

[5] Reinhardt, WilliamP., and John E. Hunter III. "Variational path optimization and upper and lower bounds to free energy changes via finite time minimization of external work." The Journal of chemical physics 97.2 (1992): 1599-1601.

[6] Ponter, Alan RS, and Jeffrey John Williams. "Work bounds and associated deformation of cyclically loaded creeping structures." (1973): 921-927.

[7] WILLIAMS, JJ. "Work Bounds and Associated Deformation of Cyclically Loaded Creeping Structures."

[8] Siirtola, Antti. "Bounds: from parameterised to finite-state verification." 2011 Eleventh International Conference on Application of Concurrency to System Design. IEEE, 2011. 
[9] Danes, Florin, and Bertrand Garnier. "Effective conductivity bounds by inserting adiabatic or is othermal surfaces." International journal of heat and mass transfer 54.15-16 (2011): 3523-3535.

「10] Carter, Peter. "Analysis of cyclic creep and rupture. Part 1: Bounding theorems and cyclic reference stres ses." International journal of pressure vessels and piping 82.1 (2005): 15-26.

[11] Layton, W. J. "Energy dissipation bounds for hear flows for a model in large eddy simulation. "Mathematical and Computer Modelling 35.13(2002): 1445-1451.

[12] Srivastava, Saket, Sudeep Sarkar, and Sanjukta Bhanja. "Es timation of upper bound of power dis sipation in QCA circuits." IEEE transactions on nanotechnology 8.1 (2008): 116-127.

[13] Laugesen, Richard S. "New dis sipated energies for the thin fluid film equation." Communications on Pure \& Applied Analysis 4.3(2005): 613.

$\lceil 14\rceil$ Bryant, Samuel J., and Benjamin B. Machta. "Energy dissipation bounds for autonomous thermodynamic cycles." Proceedings of the National Academyof Sciences 117.7 (2020): 3478-3483.

[15] Bryant, Samuel J., and Benjamin B. Machta. "Energy Dissipation Bounds in Autonomous Thermodynamic Sys tems." arXiv preprint arXiv:1903.06780 (2019).

[16] Burbury, S. H. "LVII. The second law of Thermodynamics." The London, Edinburgh, and Dublin Philosophical Magazine and JournalofScience 37.229 (1894): 574-578.

[17] Carnot, Sadi, RudolfClausius, and William Thomson Baron Kelvin. The SecondLaw ofThermodynamics. American Book Company, 1899. 